\title{
Variaciones musculares del antebrazo como factores de compresión neurovascular
}

\author{
H.F. BIANCHI \\ FACULTAD DE MEDICINA UBA \\ Correspondencia: \\ Dr. Homero F. Bianchi \\ La Pampa 2477, $4^{\circ}$ A \\ Ciudad Autónoma de Bs AS 1428 \\ Teléf: 54-11-47888173 \\ e-mail: fbianchi@fibertel.com.ar
}

$3^{a}$ Cátedra de Anatomía. Facultad de Medicina Universidad de Buenos Aires. Cátedra de anatomía del Instituto Universitario de Ciencias de la Salud de la Fundación H. A. Barceló ESPECIALISTA EN CIRUGÍA DE LA MANO

PRofesor Titular DEPARTAMENTO DE ANATOMÍA

Se lleva a cabo una revisión de variaciones musculares del antebrazo volar consideradas como factores potenciales de compresión de los nervios mediano e interóseo anterior y de la arteria ulnar, las cuales se encuentran vinculadas al flexor digitorum sublimis, al flexor pollicis longus y al pronator teres. El estudio mostró que en relación al flexor digitorum sublimis podemos describir arcadas tendinosas y fascículos accesorios, mientras que el flexor pollicis longus mostró al fascículo accesorio conocido como músculo de Gantzer, clasificado de acuerdo a su inserción distal según terminara en el flexor pollicis longus, flexor digitorum profundus o en el flexor digitorum sublimis. La variación del pronator teres consistió en el reemplazo del fascículo profundo por un fascículo tendinoso. Se relacionaron los hallazgos con los nervios y arteria mencionados, señalando su importancia en los síndromes de compresión incluyendo al de Volkmann y en los problemas derivados de la terminación del fascículo de Gantzer en el tendón del índice del flexor digitorum profundus

Palabras clave: flexor digitorum sublimis, músculo de Gantzer, variaciones musculares.
The revision of the muscular variations of the volar forearm with origin in the flexor digitorum sublimis, the flexor pollicis longus and the pronator teres considered like potential factors of compression of the median and interosseous nerves and ulnar artery is present. The study showed that in relation to the flexor digitorum sublimis we can describe tendinous arches and accessory fascicles, whereas the flexor pollicis longus showed the accessory fascicle well-known like muscle of Gantzer, classified according to its distal insertion in the flexor pollicis longus, flexor digitorum profundus and flexor digitorum sublimis. The variation of the pronator teres consisted of the replacement of the deep fascicle by a tendinous fascicle. The findings are related in relation with the mentioned nerves and artery, indicating their importance in the syndromes of compression including the Volkmann's syndrome and in the problems derived from the insertion of Gantzer's fascicle in index tendon of the flexor digitorum profundus.

Key Words: flexor digitorum sublimis, Gantzer,s muscle, muscle variations.

Rev. Iberam. Cir. Mano - Vol. 36 - Núm. 1 Mayo 2008 (8-13) 


\section{INTRODUCCIÓN}

$\mathrm{E}$ $\mathrm{s}$ reconocido el rol de las variaciones musculares del tercio superior del antebrazo volar como potenciales factores de compresión de estructuras vasculonerviosas. Esto ha motivado publicaciones sobre dichas variaciones, en especial en lo referido a la compresión del nervio interóseo anterior (Nia) por un fascículo accesorio del flexor pollicis longus (aFpl) $)^{1-12}$ descrito por Gantzer en 1813, aunque existen referencias de que Albinus los describió cien años antes ${ }^{9}$. Testut ${ }^{12}$ menciona tres tipos de inserción distal de este fascículo accesorio: en el flexor pollicis longus (Fpl), en el flexor digitorum profundus (Fdp) y en el flexor digitorum sublimis (Fds).

Existe información de hallazgos quirúrgicos de numerosos tractos o fascículos musculares del pronator teres $(\mathrm{Pt})$, al igual que estructuras vasculares ${ }^{13,14}$ que cruzan al nervio mediano $(\mathrm{Nm})$ en casos de síndrome compresivo del pronador $^{14}$ con mejoría de la sintomatología después de su sección. Presentamos las conclusiones sobre la revisión de las variedades musculares en relación con el Fpl, el Fds y el Pt que pueden constituirse en factores de compresión de elementos vasculares y neurales.

\section{MATERIAL Y MÉTODO}

El estudio se realizo en la $3^{\text {a }}$ Cátedra de Anatomía de la Facultad de Medicina de la Universidad de Buenos Aires y en la Cátedra de

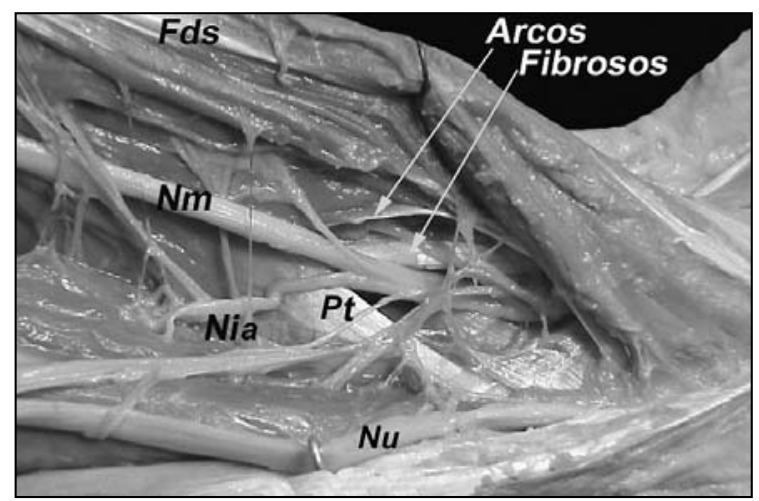

Figura 1. Fds: flexor digitorum sublimis, Pt: pronator teres con bordes tendinosos, Nm: nervio mediano, Nia: nervio interóseo anterior, $\mathrm{Nu}$ : nervio ulnar.
Anatomía de la Facultad de Medicina del Instituto Universitario de Ciencias de la Salud de la Fundación HA Barceló. Se emplearon 44 especímenes de cadáveres adultos formolados al $10 \%$, aclarados con peróxido de hidrogeno al $10 \%$. Para acceder a las estructuras se eligió un acceso medial sobre el proceso condileo medial, que se prolonga en el antebrazo $1 \mathrm{~cm}$ por dentro la proyección del hueso ulnaris hasta la muñeca, con sección de la inserción proximal de los músculos epitrocleares y su desplazamiento hacia arriba y afuera para descubrir la presencia de fascículos accesorios, y, por extensión, del Nm, y del Nia y sus ramas de división, y observar su relación, en caso de existir, con las variaciones encontradas.

\section{HALLAZGOS}

Se encontraron variaciones musculares en 37 de los 44 preparados (84\%) siendo clasificadas en tres grupos en base al músculo que origina la variedad: variaciones del Fds, del Fpl, y del Pt.

Variaciones del Fds

Totalizaron 12 preparaciones, (32.4\%), divididas en dos grupos:

\section{A1- arcadas fibrosas}

Se constataron en 7 preparaciones $(58.3 \%)$; no existió una morfología uniforme: en 2 casos se observó una arcada fibrosa gruesa (Figura 1) en el punto de penetración del Nm (28.6\%); en 3 casos una arcada fina (43\%); en 1 caso (14.2\%), una arcada múltiple (Figura 2) marcando el lugar de penetración del Nm en el Fds



Figura 2. Fds: flexor digitorum sublimis, Fdp: flexor digitorum profundus, Nia: nervio interóseo anterior, $\mathrm{Nm}$ nervio mediano, Pt: pronator teres. 


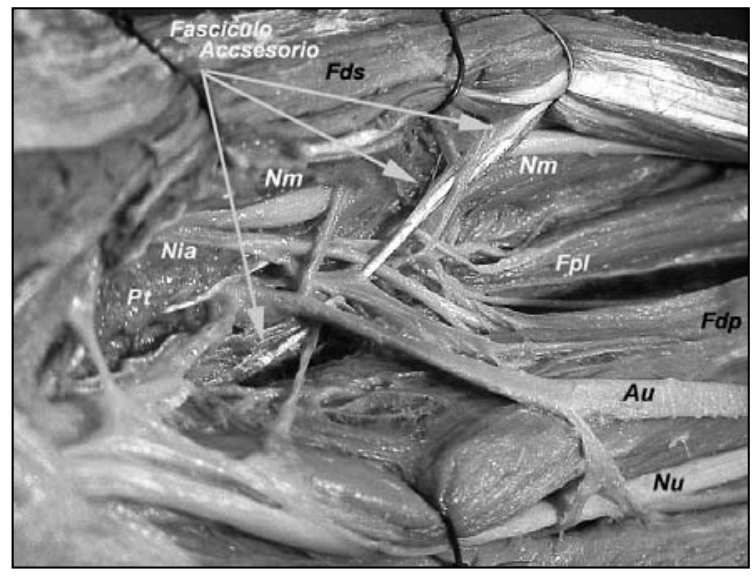

Figura 3. Fds: flexor digitorum sublimis, Fpl: flexor pollicis longus, Fdp: flexor digitorum profundus, Pt: pronator teres, Nm: nervio mediano, Nia: nervio interóseo anterior y ramas, $\mathrm{Au}$ : arteria ulnar, $\mathrm{Nu}$ : nervio lunar.

(20\%) y en el caso restante (14.2\%), una firme arcada tendinosa principal y una accesoria.

A2- fascículos accesorios (musculares y tendinosos)

Verificados en 5 preparados $(41.7 \%)$, de los cuales en 3 casos $(60 \%)$ toman inserción en el proceso coronoideo, en 1 caso (20\%) provienen del Fds (Figura 3), y en el caso restante (20\%) del epicóndilo medial. Los fascículos se encuentran por fuera de la arteria ulnar $(\mathrm{Au})$ y en relación con las ramas del Nia, entre las cuales se ubican en 2 ocasiones (40\%).

B) Músculo de Gantzer o aFpl

Se clasificaron por la forma de terminación, totalizando 25 hallazgos (67.6\%).

B1) fascículos accesorios para el Fpl

En forma aislada se encontraron en 12 casos (48\%); en 9 oportunidades el origen fue claramente la cara profunda del Fds (90\%) (Figura 4); los casos restantes tomaron origen en el epicóndilo medial. Es de notar que en los casos con origen en la cara profunda del Fds, la ubicación del aFpl fue entre el $\mathrm{Nm}$ y el Nia; en los demás casos se ubicó proximalmente medial al nervio, cruzándolo luego por delante para terminar sobre el borde medial del tendón del Fpl.

B2) fascículos accesorios para el Fpl y el Fds

La doble terminación se observo en 8 preparaciones (32\%): el origen es en el proceso coronoideo y Fds 6 casos (75\%); en 2 casos

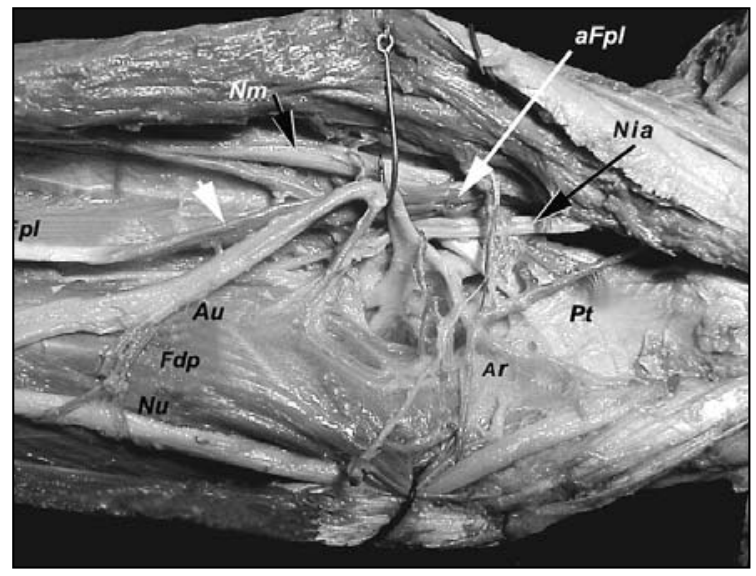

Figura 4. aFpl: accesorio flexor pollicis longus (flecha blanca larga), Fpl: flexor pollicis longus (flecha blanca corta), Fdp: flexor digitorum profundus, Pt: pronator teres, Nia: nervio interóseo anterior, (flecha negra larga), $\mathrm{Nm}$ : nervio mediano (flecha negra corta), Nu: nervio ulnar, Au: arteria ulnar, Ar: arterias recurrentes lunares.

(25\%) se origina en el epicóndilo medial, separándose desde su origen en dos fascículos que terminan en el Fds y en el Fpl; el fascículo aFpl, excepto en un caso en que se dispone medial al Nia, en el resto de las preparaciones se ubica entre ambos nervios. (Figura 5 y Figuras 6A y 6B)

B3) fascículos accesorios al Fdp

Apreciados en 5 casos (20\%), los fascículos se desprendieron de la cara profunda del Fds. Fueron variables y por lo tanto por el número pequeño no se los clasificó: 1 caso terminó en el tendón del Fdp del índice; 1 se perdió en la

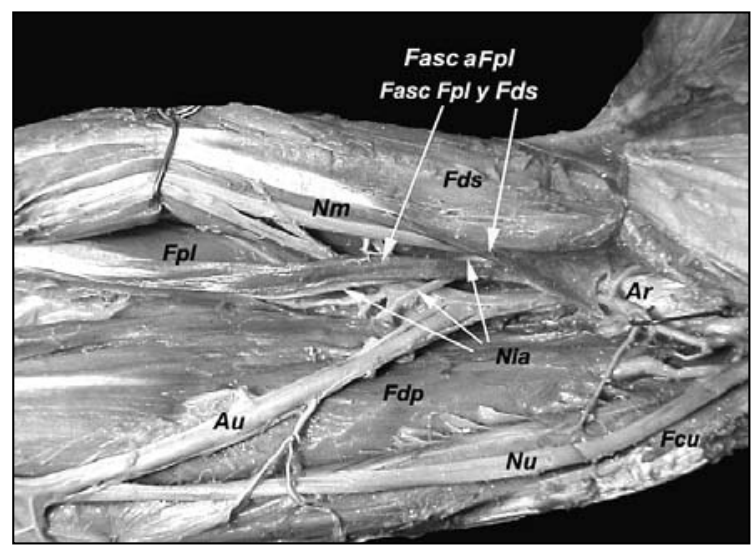

Figura 5. Fds: flexor digitorum sublimis, Fpl: flexor pollicis longus, Fdp: flexor digitorum profundus, Fcu: flexor carpi ulnaris, Au: arteria ulnar, Nu: nervio ulnar, Ar: arteria recurrente ulnar, $\mathrm{Nm}$ : nervio mediano, Nia: nervio interóseo anterior. 

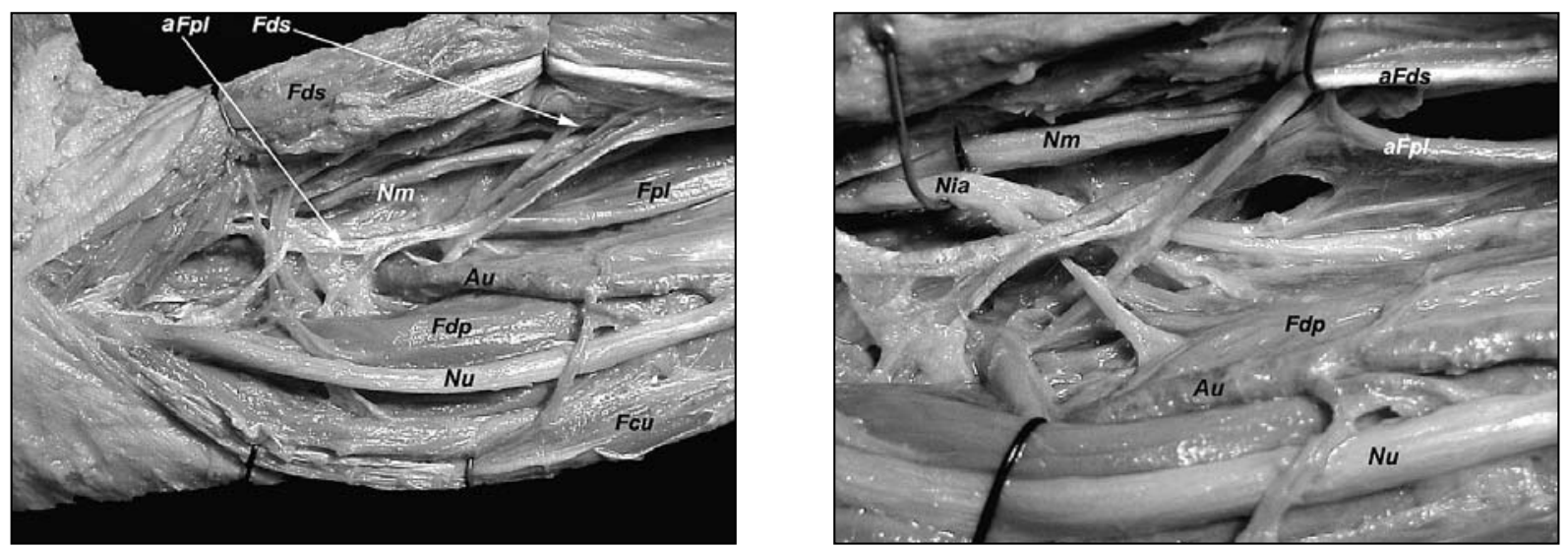

Figura 6. A: Fds: flexor digitorum sublimis, Fpl: flexor pollicis longus, Fdp: flexor digitorum profundus, Au: arteria ulnar, Nu: nervio ulnar, Fcu: flexor carpi ulnaris, Nm: nervio mediano. B: Se muestra con detalle la relación de los fascículos accesorios con las ramas del Nia.

masa muscular del Fdp; 2 casos mostraron un fascículo dividido en fascículos al Fdp y al Fpl y el restante presentó dos fascículos accesorios de origen independiente, uno al Fpl y al Fdp (Figura 7).

C) Variaciones del fascículo profundo del Pt

Diecisiete preparaciones (39\%) mostraron variación en relación a la descripción clásica, consistente en que el fascículo profundo del Pt se encuentra como una cuerda tendinosa en 12 casos $(70.5 \%$ ) (Figura 8), mientras que en el resto, solo el borde distal es tendinoso $(29.5 \%)$ (Figura 1).

\section{DISCUSIÓN}

La presencia de fascículos accesorios, bridas musculares y/o tendinosas, que unen distintos músculos entre sí, podría explicarse por el desarrollo embriológico, al tener ésta musculatura un origen común en la masa muscular epitroclear denominada grupo flexopronador de Humphrey 2, 4, 6, 9 .

La discusión seguirá el orden de los hallazgos:

Variaciones del Fds

Se estudiaron de acuerdo con su potencial de compresión sobre el $\mathrm{Nm}$ Conformaron dos grupos: las arcadas fibrosas ${ }^{11,13,14}$ y fascículos accesorios del Fds ${ }^{14}$.
Las arcadas fibrosas se encuentran en el punto de entrada del nervio mediano en el ojal muscular del Fds (Figura 4). El grosor de la arcada es variable, incidiendo esto en su potencial de compresión. No deja de ser interesante la presencia de fascículos musculares y tendinosos accesorios que toman origen en el epicóndilo medial y masa muscular próxima, y en el proceso coronoideo, terminando en la masa muscular del Fds en la proximidad de la entrada del nervio mediano, los cuales no sólo pueden comprimir a dicho nervio, sino que por las relaciones ocasionales de sus inserciones distales entre las ramas de división del Nia podrían ser causa de compresiones aisladas que actúan sobre ramas para el Fpl o el Fdp. El $\mathrm{Nm}$ no se relaciona con el aFpl, excepto cuando, como veremos, este emite el fascículo para el Fds.

En las diferentes preparaciones es común observar arterias y venas ${ }^{10,11}$ en número considerable en relación con el Nia, lo cual explica las comunicaciones de lesión neurológica por trombosis vascular. (Figuras 6A y 6B)

\section{Variaciones del Fpl}

$\mathrm{El} \mathrm{aFpl} \mathrm{ha} \mathrm{sido} \mathrm{reportado} \mathrm{en} \mathrm{alto} \mathrm{porcenta-}$ je por todos los autores consultados, con variaciones entre el 33 y el $89 \%$, siendo una de las causas mencionadas como causa de la compresión del Nia. Nuestra estadística arrojo cifras del $67.6 \%$, por lo cual compartimos el concepto de presencia habitual por ellos sostenido. 


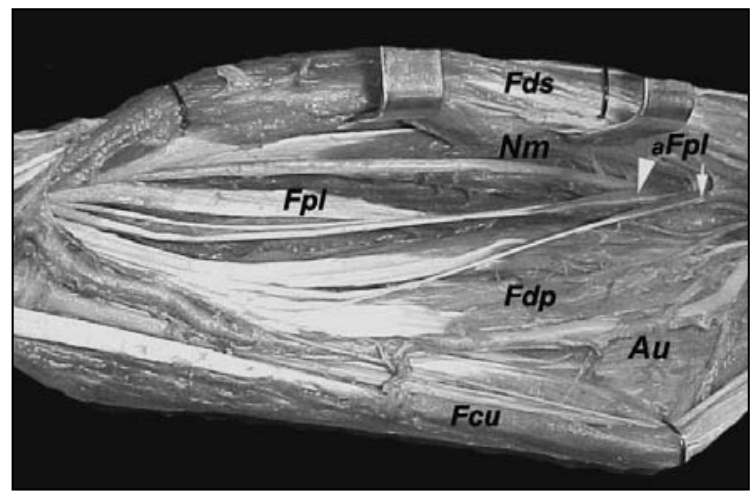

Figura 7. Fpl: flexor pollicis longus, Fds: flexor digitorum sublimis, Fdp: flexor digitorum profundus, Fcu flexor carpi ulnaris, aF pl: accesorio del flexor pollicis longus, Au: arteria ulnar, Nm: nervio mediano. El triangulo señala el aFpl a Fpl y la flecha el aFpl al Fdp.

Como origen se han citado el epicóndilo medial $^{1-6,10-12}$, el proceso coronoideo ${ }^{1,2,6}$, el epicóndilo medial y en el proceso coronoideo ${ }^{1,3}$, el epicóndilo medial asociado al origen común de los músculos flexores del antebrazo volar ${ }^{3,6}$. El origen en el Fds ${ }^{3,4}$ es para Jones y Abrahms ${ }^{4}$ el origen habitual, opinión que compartimos.

Los hallazgos de accesorios de Fpl se agruparon según su terminación distal, ya que se entendió que las mismas no eran más que distintas expresiones de una misma variedad con destino diferente, interpretando a Testut ${ }^{12}$ quien menciona que tanto el Fds, el Fdp y el Fpl pueden recibir fascículos accesorios independientes entre sí, lo cual en los especímenes resulto cierto para los destinados al Fpl y al Fdp, con una ligera diferencia en relación al Fds, en donde el fascículo se muestra como una división del aFpl.

Para incluir a los aFdp como aFpl, se tuvo en cuenta la fusión entre estos grupos musculares, común en los primates, considerando su presencia como una persistencia de la mis$\mathrm{ma}^{4}, 10,12$. En nuestra serie el origen de estos fascículos, de acuerdo con lo reportado por Jones y Abrahms ${ }^{4}$ fue la cara profunda del Fds y no el proceso coronoideo o condíleo medial como se ha mencionado.

Esta disposición resulta en alteraciones del tendón del Fpl en forma de sinovitis o roturas de tenorrafias del flexor profundo del índice después de reparaciones por lesiones traumáticas

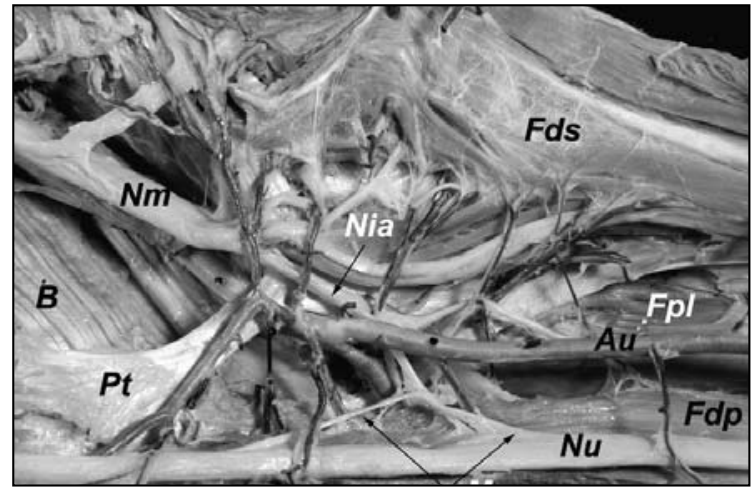

Figura 8. B: brachialis, Fds: flexor digitorum sublimis, Fpl: flexor pollicis longus, Fdp: flexor digitorum profundus, Nm: nervio mediano, Nia: nervio interóseo anterior, Au: arteria ulnar, Nu: nervio ulnar, Mg: comunicación de Martin-Grüber (flechas negras), Pt: fascículo tendinoso del pronator teres.

del mismo, y la pérdida de la independencia entre los dedos índice y pulgar.

En relación con el aPl, además de las compresiones del Nia y del Nm, Kaplan ${ }^{3,6,11}$ describió la retracción del pulgar en las fracturas de codo y se ha mencionado su alargamiento en parálisis espásticas y su liberación en casos de síndromes de Volkmann².

La posición del Nia en relación a los fascículos accesorios es posterior en relación a los aFdp (Figura 7), tal como lo señalan Jones y Abrahms ${ }^{4}$, excepto 2 casos en que es medial. En lo que se refiere al aFpl, las opiniones difieren, ya que se ha ubicado al Nia, anterior ${ }^{1}$, posterior $^{2-6}$, lateral $^{5,6}$ o lateroposterior ${ }^{5}$. Se deduce que es la posición posterior es la que lo pone en peligro de ser comprimido en forma total o parcial $^{11}$. En la serie actual la posición fue posterior al aFpl (Figura 4) salvo en 1 caso.

Compartimos $^{15}$ la opinión de Spinner ${ }^{10}$ en que el Nia es el origen principal de la comunicación de Martin-Grüber (Figura 8) por lo cual su compresión puede acompañarse de alteraciones funcionales en el territorio cubital. Por último no podemos dejar de considerar la compresión simultánea del Nm y del Nia cuando el aFpl emite el fascículo para el Fds.

\section{Variaciones del Pt}

En relación con el Pt, la variación está en el fascículo profundo, pero a diferencia de la concepción clásica $^{1,12}$, no es su ausencia lo que ca- 
racterizó los hallazgos sino su reemplazo por una cuerda tendinosa ${ }^{13}$, o bien un fascículo muscular con un borde cortante tendinoso, generalmente el inferior, lo que lo convierte en un factor potencial de compresión tanto del $\mathrm{Nm}$ y del $\mathrm{Nia}^{8}$ como de la $\mathrm{Au}$, a tener en cuenta en los síndromes compresivos en lo que a la arteria en particular se refiere.

Luego de evaluar los hallazgos nos surge una pregunta: ¿es posible que en condiciones normales se produzcan compresiones nerviosas por la acción de estos músculos accesorios? La respuesta a priori es que no, merced a la alta frecuencia de hallazgos y la baja incidencia de las mismas.

Pero están dadas las condiciones para que bajo determinadas actividades que produzcan movimientos repetidos ${ }^{14}$, ya sean deportivas, la- borales o profesionales, ocasionen que los elementos vasculonerviosos sean presionados por las estructuras accesorias determinando la aparición de los síntomas pertinentes.

\section{CONCLUSIONES}

Existen en el antebrazo un número considerable de variedades musculotendinosas en relación con los músculos Fds, Fpl y Pt, las cuales pueden ser consideradas como factores potenciales de compresión de estructuras neurovasculares. La terminación del aFpl en el Fdp, puede ser la causa de la pérdida de la individualidad de la función del índice y pulgar y del fracaso de tenorrafias del dedo índice entre las varias consecuencias de su presencia.

\section{BIBLIOGRAFÍA}

1. Al-Qattan MM. Gantzer's muscle, an anatomical study of the accessory head of the flexor pollicis muscle, $\mathrm{J}$ Hand Surg Br, 1996; 21: 269-70.

2. Hemmady MV, Subramanya AV, Metha IM. Occasional head of flexor pollicis longus muscle: a study of its morphology and clinical significance, J Postgrad Med, 1993; 39: 14-16.

3. Ho CS, Chung IH, Koh KS. Anatomical study of the accessory head of the flexor pollicis longus and the anterior interosseous nerve in asians. Clin Anat, 2000; 13: 434-8.

4. Jones M, Abrahms PH. Incidence and morphology of accessory heads of the flexor poIlicis longus and flexor digitorum profundus (Gantzer's muscles). J Anat, 1997; 191: 451-5.

5. Mahakkanukrauh $P$, Surin $P$, Ongkana N, et al. Prevalence of accessory head of flexor pollicis longus muscle and its relation to anterior interosseous nerve in Thai population. Clin Anat, 2004; 17: 631-5.

6. Mangini U. Flexor pollicis longus muscle, its morphology and clinical significance. J Bone Joint Surg Am, 1960; 42: 467-70.

7. Nagano A. Spontaneous anterior interosseous nerve palsy. $\mathrm{J}$ Bone Joint Surg Br, 2003, 8: 313-8.

8. Rask MR. Anterior interosseous nerve entrapment (Kiloh Nevin syndrome). Clin Orthop, 1979; 142: 177-81.

9. Shirali HM, Branovacki G, Gonzalez M. The flexor pollicis longus and its relation to the anterior and posterior interosseous nerves, J Hand Surg $\mathrm{Br}$, 1998; 23: 170-2.

10. Spinner M. The anterior interosseous nerve syndrome. $J$
Bone Joint Surg Am, 1970; 52 : 84-94.

11. Tabib W, Aboufarah F, Asselineau $A$. Compression du nerf interosseux anterieur par le muscle de Gantzer. Chir Main, 2001; 20, 241-6.

12. Testut L. Les anomalies muscularies chez l'homme. Paris: Mason Ed. 1884.

13. Gunther SF, DiPasquale D, Martin R. The internal anatomy of the median nerve in the region of the elbow. $\mathrm{J}$ Hand Surg Am, 1992; 17: 648-56.

14. Hartz ChR, Linscheid RL, Gramse RR, et al. The pronator teres syndrome compressive neuropathy of the median nerve. J Bone Joint Surg Am, 1981; 63: 885-9.

15. Bianchi $\mathrm{H}$, Anastomosis mediano cubital en el antebrazo, Semana Médica, 1982; 161: 103-106. 